\title{
Message from General Co-chairs and Program Co-chairs
}

Welcome to Huangshan and to the joint 2010 International Conference on Computational Sciences and Optimization (CSO2010), held at Huangshan, Anhui, China, May 28-31, 2010. CSO2010 builds on the success both the first conference held at Hong Kong, China, August 1-3, 2008 and the second conference held at Sanya, Hainan, China, April 24-26, 2009. Encouraged by the successful experience and feedbacks from the participants, this year the conference is held at Huangshan, Auhui, China.

Computational Sciences and Optimization are two important disciplines in modern sciences with many applications ranging from applied sciences, engineering, management, finance, and economics, to arts, humanities, and social sciences. Currently some new challenges arise in the modeling of complex systems, sophisticated algorithms, advanced scientific and engineering computing and associated problem-solving environments. Because the solutions of complex problems must cope with the tight timing schedule, powerful computational algorithms and complex optimization techniques are inevitable for solving these practical problems.

In order to strengthen academic exchanges and discussions on the fields of computational sciences and optimization, The Third International Joint Conference on Computational Sciences and Optimization (CSO2010) including The 2010 International Symposium on Applied Computing and Computational Sciences (ACCS2010) and The 2010 International Symposium on Optimization Theory, Methods and Applications (OTMA2010) will be held on May 28-31, 2010 in Huangshan (Yellow) Mountain, Anhui Province, China. It provides a four-day forum on Computational Sciences and Optimization Sciences. The theme of the plenary session is "Principles of Practice in Computational Sciences and Optimization" and the following keynote speakers are invited to further explore these topics: Wolfgang Gentzsch (DEISA Distributed European Initiative for Supercomputing Applications, Germany), Sy-Ming Guu (Yuan Ze University, Taiwan), Gang Kou (University of Electronic Science and Technology of China, China), ChoLi Wang (the University of Hong Kong, China), Laurence T. Yang (St Francis Xavier University, Canada), Ning Zhong (Maebashi Institute of Technology, Japan). The General Co-chairs of CSO2010 would like to thank all the keynote speakers for their innovative and inspiring speeches delivered in the conference.

Concurrent sessions and a poster session will cover a wide range of topics and issues, including both contributed papers and special sessions/workshops developed on specific topics, all with a central focus of Applied Computing, Computational Sciences, Optimization Theory, Methods and Applications. This year, we also have four workshops that complemented CSO2010 program with contributions for specific topics. The workshops include the 2nd International Workshop on Computational Transportation Science (CTS2010) (organized by Qiong Tian, Hu Shao, and Tianliang Liu), the 2nd International Workshop on Intelligent Computing in Meteorological Applications (ICMA2010) (organized by Jin Long and Wu Jiansheng), the 2nd International Workshop on Intelligent Information Processing (IIP2010) (organized by Wenqian Shang and Haibin Zhu), the 2010 IEEE International Workshop on HPC and Grid Applications (IWHGA2010) (Organized by Yingwen Song). The Program Co-chairs of CSO2010 would like to thank all the workshops chairs for their excellent works and effort in organizing these workshops.

The CSO 2010 joint conference will provide an idea-exchange and discussion platform for the world's researchers and academia, where internationally recognized researchers and practitioners share state-ofthe-art research results, cutting-edge information, address the hottest issue in computational science and optimization, explore new computational technologies, exchange and build upon new ideas. And meantime the joint conference will also provide researchers and practitioners an opportunity to highlight innovative research directions, and a growing number of novel applications, and to explore new approaches for future developments. 
CSO2010 received more than 700 submissions (including main track papers and workshop papers) from over 20 countries and regions. All submitted papers have to go through a rigorous reviewing process. Each submission was reviewed by at least two independent reviewers in a standard peer-review process. Papers belonged to three categories: regular papers of 5 pages, short papers of 4 pages and poster papers of 2 pages. After rigorous peer-review, we finally select 229 papers (acceptance rate $32 \%$ ) for publication. We emphases that our policy is to guarantee the high-quality of the accepted papers and at the same time encourage more people to participate in the conference.

This year, CSO2010 is co-sponsored by Chinese Academy of Sciences (CAS), China; Department of Management Science of National Natural Science Foundation of China (NSFC), China; Academy of Mathematics and Systems Science of CAS, Institute of Systems Science of CAS; Centre for Forecasting Science (CEFS) of CAS, China; Key Laboratory of Management, Decision and Information Systems (MADIS) of CAS, China; International Institute for Decision Sciences (IIDS), Hong Kong; and Painting and Calligraphy Institute, Beijing Xueze Academy, China. Meantime, CSO 2010 is technically cosponsored by IEEE Computer Society, USA; College of Business of City University of Hong Kong, Hong Kong; System Engineering Society of China (SESC), China; IEEE Computer Society Technical Committee on Intelligent Informatics (TCII), USA; IEEE Computer Society Technical Committee on Scalable Computing (TCSC), Canada; Beijing Operation Research Society, China; Technical Committee on Decision Science of Operation Research Society of China, China; Technical Committee on Management Science and Engineering of Chinese Society of Management Modernization, China; Technical Committee on Financial System Engineering of System Engineering Society of China, China. Their sponsorships support the success of conference.

CSO2010 would not have been successful without the support of many people and organizations. First and foremost, we would like to thank all the authors for submitting their papers to the conference, for their presentations and discussions during the conference. We would like to express our most sincere gratitude to Program Committee members and our professional reviewers, who carried out the most difficult work by carefully evaluating the submitted papers. We would like to give special thanks to the conference sponsors. Last but not least, we would like to thank all conference participants for their contribution and support. We hope that all participants can take this opportunity to share and exchange ideas with one another and enjoy CSO2010.

K. K. Lai, City University of Hong Kong, Hong Kong Yingwen Song, Applied Science \& Technology Research Institute, Hong Kong

Shouyang Wang, Chinese Academy of Sciences, China

General Co-Chairs of CSO2010

W. K. Ching, The University of Hong Kong, Hong Kong
Hai Jin, Huazhong University of Science and Technology, China
Jianping Li, Chinese Academy of Sciences, China
S. K. Mishra, Banaras Hindu University, India
Lean Yu, Chinese Academy of Sciences, China
Program Co-Chairs of CSO2010

